\title{
High-K Isomers in Light Superheavy Nuclei by PNC-CSM method
}

\author{
Xiao-Tao $\mathrm{He}^{1, *}$ \\ ${ }^{1}$ College of Material Science and Technology, Nanjing University of Aeronautics and Astronautics, \\ Nanjing 210016, China
}

\begin{abstract}
The high- $K$ isomeric states in light superheavy nuclei around $A=$ 250 mass region are investigated by the Cranked Shell Model (CSM) with pairing treated by a Particle-Number Conserving (PNC) method. With including the higher-order deformation $\varepsilon_{6}$, both of the high- $K$ multi-particle state energies and the rotational bands in ${ }^{254}$ No and $N=150$ isotone are reproduced well. The isomeric state energies and the microscopic mechanism of kinematic moment of inertia variations versus rotational frequency are discussed. The irregularity of the two-neutron $K^{\pi}=8^{-}$state band at $\hbar \omega \approx 0.17$ in ${ }^{252}$ No is caused by the configuration mixing with the two-proton $K^{\pi}=8^{-}$band. .
\end{abstract}

\section{Introduction}

In recent decades, intensive studies of the in-beam and decay spectroscopic have been performed on the light well-deformed superheavy nuclei around $A=250$ mass region. Increasing experimental spectroscopic data have been reported [1, 2]. Moreover, $A=250$ region is one of the typical mass region for presence of the high $-K$ isomers. Numerous isomers (mainly two-particle states) are identified now (see Table 1 in Ref.[3]). Beside the twoparticle states, four-particle states are also observed, like the 2.928(3) MeV 184(2) $\mu$ s isomer in ${ }^{254} \mathrm{No}$ [4] and $247(73) \mu$ s isomer in ${ }^{254} \mathrm{Rf}$ [5]. These experimental data provide valuable informations to explore the structure of the light superheavy nuclei and to test the nuclear theoretical models. Moreover, through the investigations of single-particle structure of the light superheavy nuclei, one can predict more precisely the next spherical magic number beyond ${ }^{208} \mathrm{~Pb}$. This is crucial for producing the superheavy elements. In the present work, the high- $K$ multi-particle state bands in ${ }^{254}$ No and the $K^{\pi}=8^{-}$isomeric bands observed systematically in $N=150$ isotone are investigated by Cranked Shell Model with pairing correlation treated by Particle-Number-Conserving method.

\section{Theoretical method}

The cranked shell model hamiltonian of an axially symmetric nucleus in the rotating frame is $H_{\mathrm{CSM}}=H_{0}+H_{\mathrm{P}}=\sum_{n}\left(h_{\mathrm{Nil}}-\omega j_{x}\right)_{n}+H_{\mathrm{P}}(0)+H_{\mathrm{P}}(2)$, where $h_{\mathrm{Nil}}$ is the Nilsson Hamiltonian, $-\omega j_{x}$ is the Coriolis force with the cranking frequency $\omega$ about the $x$ axis (perpendicular to the nuclear symmetry $z$ axis). $H_{\mathrm{P}}$ is the pairing including monopole and quadrupole pairing

*e-mail: hext@nuaa.edu.cn 
Table 1. Multi-particle states predicted by the PNC-CSM calculations.

\begin{tabular}{ccccl}
\hline \hline nuclei & $K^{\pi}$ & Configuration & $E_{x}(\mathrm{MeV})$ & $E_{x}^{\text {exp }}(\mathrm{MeV})$ \\
\hline${ }^{250} \mathrm{Fm}$ & $8^{-}$ & $v 9 / 2^{-}[734] \otimes v 7 / 2^{+}[624]$ & 1.213 & 1.199 \\
${ }^{252} \mathrm{No}$ & $8^{-}$ & $v 9 / 2^{-}[734] \otimes v 7 / 2^{+}[624]$ & 1.139 & 1.254 \\
${ }^{254} \mathrm{No}$ & $3^{+}$ & $\pi 7 / 2^{-}[514] \otimes \pi 1 / 2^{-}[521]$ & 1.154 & 0.988 \\
${ }^{254} \mathrm{No}$ & $8^{-}$ & $\pi 9 / 2^{+}[624] \otimes \pi 7 / 2^{-}[514]$ & 1.272 & 1.297 \\
${ }^{254} \mathrm{No}$ & $10^{+}$ & $v 9 / 2^{-}[734] \otimes v 11 / 2^{-}[725]$ & 2.526 & 2.013 \\
${ }^{254} \mathrm{No}$ & $16^{+}$ & $\left\{v 9 / 2^{-}[734] \otimes v 7 / 2^{+}[613]\right.$ & 3.213 & 2.928 \\
& & $\left.\pi 9 / 2^{+}[624] \otimes \pi 7 / 2^{-}[514]\right\}$ & & \\
\hline \hline
\end{tabular}

correlations. $H_{\mathrm{CSM}}$ is diagonalized directly in a truncated Cranked Many-Particle Configuration (CMPC) (Fock) space. Therefore, particle number is conserved and Pauli blocking effects are taken into account exactly.

The Nilsson parameters $(\kappa, \mu)$ which are optimized to reproduce the experimental level schemes for heavy nuclei around $A=250$ mass region in Refs. [6, 7] are used. The axially symmetric deformation parameters $\varepsilon_{2,4,6}$ are taken from Refs. [7, 8]. The CMPC space is constructed in the proton $N=4,5,6$ and neutron $N=6,7$ shells. The dimensions of the CMPC space are about 1000 and the corresponding effective monopole and quadrupole pairing strengths are $G_{0}=0.25 \mathrm{MeV}$ and $G_{2}=0.02 \mathrm{MeV}$ for both of proton and neutron for all the studied nuclei.

\section{High $-K$ multi-particle states}

Multi-particle state configurations and energies in ${ }^{254}$ No and in the $N=150$ isotone calculated by the PNC-CSM are listed in Table 1. A better reproduction of the experimental state energies will be obtained by including of $\varepsilon_{6}$ deformation. The $K^{\pi}=8^{-}$isomer state is observed systematically in this mass region. Unlike the $K^{\pi}=8^{-}$isomer in the $N=150$ isotones, whose configuration is accepted as two-neutron $v 9 / 2^{-}[734] \otimes v 7 / 2^{+}[624]$ state in the literature, the configuration of the observed $K^{\pi}=8^{-}$isomer state in ${ }^{254}$ No is in dispute up to now. In the PNC-CSM calculation, the lowest $8^{-}$state in ${ }^{254}$ No has the two-proton $\pi 9 / 2^{+}[624] \otimes \pi 7 / 2^{-}[514]$ configuration with energy $1.272 \mathrm{MeV}$, which reproduces very well the experimental value 1.297(2) MeV.

The experimental kinematic moment of inertia $J^{(1)}$ among the ground state band (GSB) and the $K^{\pi}=8^{-}$state band in ${ }^{254} \mathrm{No}$ and in $N=150$ isotone $\left({ }^{252} \mathrm{No}\right.$ and $\left.{ }^{250} \mathrm{Fm}\right)$ are compared with the PNC-CSM calculations in Figure 1. The theoretical results reproduce the experimental data well. The authors in Ref. [9] claimed that there is an irregularity of the experimental dynamical moments of inertia $J^{(2)}$ at $\hbar \omega \approx 0.17 \mathrm{MeV}(\operatorname{spin} I \sim 17 \hbar$ ) of the $K^{\pi}=8^{-}$band in ${ }^{252}$ No (see Figure 6 in Ref.[9]), and they suggested that this irregularity is due to the band crossing with a $K^{\pi}=2^{-}$octuple band. The PNC-CSM calculations show that the behaviors of $J^{(1)}$ for two-neutron $K^{\pi}=2^{-}$(or $7^{-}$), $v 9 / 2^{-}[734] \otimes v 5 / 2^{+}[622]$ state at $\hbar \omega \geq 0.15 \mathrm{MeV}$ is similar with that for $K^{\pi}=8^{-}$state. But its energy is too high. This might imply that the irregularity in MoI is not from the crossing with the two-neutron $K^{\pi}=2^{-}$(or $7^{-}$) state. The $J^{(1)}$ for the two-proton $K^{\pi}=8^{-}, \pi 9 / 2^{+}[624] \otimes \pi 7 / 2^{-}[514]$ state shows a slight upbending at $\hbar \omega \approx 0.17 \mathrm{MeV}$, and the gradual increase afterwards can reproduce the experimental variations well. Thus, if the assumption that the irregularity of $\mathrm{MoI}$ at $\hbar \omega \geq 0.17 \mathrm{MeV}$ coming from band-crossing is correct, it might result from the crossing with the two-proton $\pi 9 / 2^{+}[624] \otimes \pi 7 / 2^{-}[514]$ configuration, which itself is mixed with $\pi 9 / 2^{+}[624] \otimes \pi 1 / 2^{-}[521]$ configuration. 


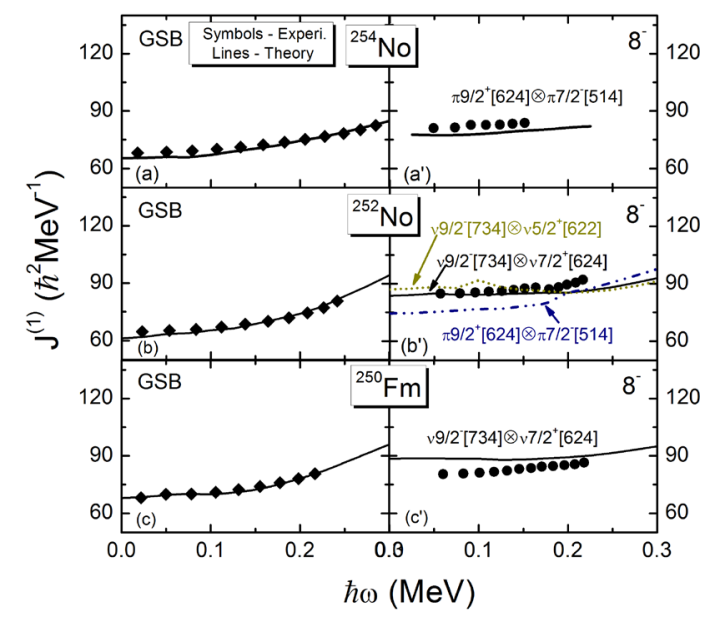

Figure 1. (Color online) Kinematic moment of inertia $J^{(1)}$ versus rotational frequency $\hbar \omega$ of the ground state bands [(a),(b),(c)] and the $K^{\pi}=8^{-}$state bands $\left[\left(\mathrm{a}^{\prime}\right),\left(\mathrm{b}^{\prime}\right),\left(\mathrm{c}^{\prime}\right)\right]$ in ${ }^{254}$ No and in $N=150$ isotone $\left({ }^{252} \mathrm{No}\right.$ and $\left.{ }^{250} \mathrm{Fm}\right)$. Theoretical calculations are denoted by lines and experiment data are denoted by solid symbols.

\section{Summary}

In summary, the Cranked Shell Model with pairing correlations treated by a Particle-Number Conserving method is used to study the multi-particle states observed in ${ }^{254} \mathrm{No}$ and in the $N=$ 150 isotone. Calculations including high-order deformation $\varepsilon_{6}$ lead to a better reproduction of the experimental data. The irregularity of the two-neutron $K^{\pi}=8^{-}$state band in ${ }^{252}$ No is analysed together with other low-lying two-particle state bands. The abnormal behavior at $\hbar \omega \approx 0.17$ of the MoI is caused by the band cross with two-proton $K^{\pi}=8^{-}$band.

\section{Acknowledgments}

This work was supported by the National Natural Science Foundation of China under Grant No. 11775112 and 11275098.

\section{References}

[1] R.D. Herzberg, P.T. Greenlees, Prog. Part. Nucl. Phys. 61, 674 (2008)

[2] R.D. Herzberg, J. Phys. G: Nucl. Part. Phys. 30, R123 (2004)

[3] F. Kondev, G. Dracoulis, T. Kibédi, At. Data Nucl. Data Tables 103-104, 50 (2015)

[4] R. Clark, K. Gregorich, J. Berryman, M. Ali, J. Allmond, C. Beausang, M. Cromaz, M. Deleplanque, I. Dragojević, J. Dvorak et al., Phys. Lett. B 690, 19 (2010)

[5] H.M. David, J. Chen, D. Seweryniak, F.G. Kondev, J.M. Gates, K.E. Gregorich, I. Ahmad, M. Albers, M. Alcorta, B.B. Back et al., Phys. Rev. Lett. 115, 1 (2015)

[6] Z.H. Zhang, J.Y. Zeng, E.G. Zhao, S.G. Zhou, Phys. Rev. C 83, 1 (2011)

[7] Z.H. Zhang, X.T. He, J.Y. Zeng, E.G. Zhao, S.G. Zhou, Phys. Rev. C 85, 1 (2012)

[8] P. Möller, J. Nix, At. Data and Nucl. Data Tables 59, 185 (1995)

[9] B. Sulignano, C. Theisen, J.P. Delaroche, M. Girod, J. Ljungvall, D. Ackermann, S. Antalic, O. Dorvaux, A. Drouart, B. Gall et al., Phys. Rev. C 86, 1 (2012) 